\title{
Cecal Microbiota Modulates Fat Deposition in Muscovy Ducks
}

\section{OPEN ACCESS}

Edited by:

Rajesh Jha

University of Hawaii at Manoa,

United States

Reviewed by:

Ahmed Mohamed Fouad,

Cairo University, Egypt

Sugiharto Sugiharto,

Diponegoro University, Indonesia

*Correspondence:

Hua Yang

yanghua@mail.zaas.ac.cn

Yingping Xiao

ypxiaozj@hotmail.com

Specialty section:

This article was submitted to Animal Nutrition and Metabolism,

a section of the journa

Frontiers in Veterinary Science

Received: 07 October 2020

Accepted: 11 March 2021

Published: 31 March 2021

Citation:

Lyu W, Liu X, Lu L, Dai B, Wang W,

Yang $\mathrm{H}$ and Xiao $Y$ (2021) Cecal Microbiota Modulates Fat Deposition in Muscovy Ducks.

Front. Vet. Sci. 8:609348 doi: 10.3389/fvets.2021.609348

\section{Wentao Lyu ${ }^{1}$, Xiuting Liu ${ }^{1}$, Lizhi Lu ${ }^{2}$, Bing Dai ${ }^{3}$, Wen Wang ${ }^{1}$, Hua Yang ${ }^{1 *}$ and Yingping Xiao ${ }^{1 *}$}

\begin{abstract}
'State Key Laboratory for Managing Biotic and Chemical Threats to the Quality and Safety of Agro-Products, Institute of Quality and Standard for Agro-Products, Zhejiang Academy of Agricultural Sciences, Hangzhou, China, ${ }^{2}$ Institute of Animal Husbandry and Veterinary Science, Zhejiang Academy of Agricultural Sciences, Hangzhou, China, ${ }^{3}$ College of Animal Sciences \& Technology, Zhejiang A \& F University, Hangzhou, China
\end{abstract}

Ducks with the same genetic background vary greatly in their adiposity phenotypes. The gut microbiota plays an essential role in host physiological development and metabolism including fat deposition. However, the association of the gut microbiota with the lipogenic phenotype of ducks remains unknown. In this study, we investigated the cecal microbiota of adult Muscovy ducks and the correlation of the cecal microbiota with fat phenotypes. A total of 200 Muscovy ducks were selected from a population of 5,000 Muscovy ducks to record their abdominal fat weight and collect their cecal contents after being slaughtered and defeathered. The cecal contents were subjective to DNA isolation and 16S rRNA gene sequencing. The results were sorted according to the percentage of abdominal fat and the top 20\% $(n=40)$ and the bottom $20 \%$ $(n=40)$ were set as the high and low groups, respectively. Our results indicated that in the cecum of Muscovy ducks, Bacteroidetes, Firmicutes, and Fusobacteria were the predominant phyla while Bacteroides, Oscillospiraceae_uncultured, Parabacteroides, and Bacteroidales_norank were the top 4 dominant genera. Abdominal fat weight $(18.57 \sim 138.10 \mathrm{~g})$ and percentage of abdominal fat (1.02 27.12\%) were significantly correlated $\left(R^{2}=0.92, P<0.001\right)$. Although the lipogenic phenotypes of ducks had a significant difference $(P<0.05)$, the $\alpha$-diversities of the high and low groups were not significantly different $(P>0.05)$. Nevertheless, after random forest analysis, we identified two genera, Treponema and Ruminococcus_torques_group, that were significantly associated with fat deposition in Muscovy ducks. In addition, the abundances of Treponema and Ruminococcus_torques_group gave a significantly negative and positive association with abdominal fat weight, respectively $(P<0.05)$. Ducks with a low level of Treponema exhibited a tendency toward a high percentage of abdominal fat $(P<0.01)$, while the percentage of abdominal fat in ducks with high Ruminococcus_torques_group abundance tended to be higher than that in ducks with low Ruminococcus_torques_group abundance $(P<0.01)$. These findings could provide the basic data on the cecal microbiota in Muscovy ducks as well as a theoretical foundation to limit the fat deposition by modulating the gut microbiota in the duck industry.

Keywords: abdominal fat deposition, cecal microbiota, muscovy ducks, Ruminococcus_torques_group, Treponema 


\section{INTRODUCTION}

The global prevalence of obesity and obesity-related diseases has been increasing rapidly (1). Duck meat provides another protein source mainly in Asia, with a high content of essential unsaturated fatty acids, and it is popular in China. However, modern rearing conditions may lead to various developmental and metabolic disorders (2). Excessive abdominal fat deposition is a waste of dietary energy, which is more preferentially deposited into edible parts such as muscle (3). Additionally, considering health, people are consciously limiting the intake of lipids, so consumers would prefer duck meat with high intramuscular content instead of abdominal fat (4). In the poultry industry, approximately 3 million tons of abdominal fat are discarded each year worldwide, leading to a $\$ 2.7$ billion loss (5).

Similar to in humans and other farm animals, trillions of commensal microorganisms are harbored in the duck gastrointestinal tract, functioning to facilitate the digestion and absorption of nutrients and energy from the diet $(6,7)$. Therefore, it is possible to develop strategies to enhance physiological status, including growth performance, by altering the gut microbiota. Fecal microbiota transplantation experiments, transferring fecal microbiota from obese humans, mice or pigs to germ-free or antibiotic-treated mice, boosted fat deposition (8-10). This finding indicates that the gut microbiota plays a vital role in the fat metabolism. Furthermore, two microbial taxa, Methanobrevibacter and Mucispirillumschaedleri were identified from broiler cecal content with a significant correlation with fat deposition (5).

Muscovy ducks (Cairinamoschata) have been domesticated for centuries and are characterized by strong-tasting meat with leanness and tenderness (11). Muscovy ducks are excellent meat ducks with high nutritional value, exhibiting high content of unsaturated fatty acids, various amino acids, vitamin E, vitamin $B$, and trace elements such as iron, copper, zinc, etc. Most investigations on Muscovy ducks involve reovirus and parvovirus (12-16). A recent study revealed that variability in fat accumulation was correlated with the cecal microbiota in chickens (5). The authors also found two microbial taxa, Methanobrevibacter and Mucispirillumschaedleri, with a strong association with fat deposition. A further understanding of the gut microbiota is needed to control the abdominal fat deposition by regulating the gut microbiota. Little is known about the gut microbiota composition in Muscovy ducks. The present study aimed to study the cecal microbial composition in Muscovy ducks and associate the relative contribution of the cecal microbiota to fat-related phenotypes. In the present study, we performed 16S rRNA gene sequencing on cecal content samples from 200 Muscovy ducks to investigate the commensal bacterial composition in the cecum of Muscovy ducks. We found three predominant phyla, Bacteroidetes, Firmicutes, and Fusobacteria, and four most abundant genera, Bacteroides, Oscillospiraceae_uncultured, Parabacteroides, and Bacteroidales_norank. After random forest analysis, we identified two genera, Treponema and Ruminococcus_torques_group, that were negatively and positively correlated with fat-related phenotypes, respectively. The findings of this study will provide
TABLE 1 | Composition and nutrient levels of diets.

\begin{tabular}{lcc}
\hline Ingredient (\%) & Starter & Finisher \\
\hline Corn & 58.90 & 56.50 \\
Soybean meal & 28.00 & 20.00 \\
Wheat & 7.27 & 18.00 \\
Soybean oil & 2.05 & 1.85 \\
Sodium carbonate & 1.14 & 1.16 \\
Dicalcium phosphate & 0.68 & 0.64 \\
Lysine & 0.285 & 0.315 \\
Methionine & 0.265 & 0.235 \\
NaCl & 0.40 & 0.24 \\
Choline chloride & 0.06 & 0.06 \\
Vitamin and trace mineral premix & 1.00 \\
Calculated nutrients levels (\%) & & 1.00 \\
Metabolizable energy, MJ/kg & 12.12 & \\
Crude protein & 20.50 & 11.58 \\
Calcium & 0.86 & 16.50 \\
Phosphorus & 0.53 & 0.95 \\
Lysine & 0.89 & 0.52 \\
Methionine & 0.51 & 0.92 \\
\hline & & 0.49 \\
\hline
\end{tabular}

a The premix provided per kilogram of total diet: vitamin A, 10,000 IU; vitamin D3, $2100 \mathrm{IU}$; vitamin E, $15 \mathrm{U}$; vitamin K3, $1 \mathrm{mg}$; vitamin B1, $2 \mathrm{mg}$; vitamin B2, $4 \mathrm{mg}$; vitamin B6, 3 mg; vitamin B12, $0.005 \mathrm{mg}$; nicotinic acid, $40 \mathrm{mg}$; pantothenic acid, $10 \mathrm{mg}$; folic acid, I mg; biotin, $0.3 \mathrm{mg}$; choline, 2,000 mg; Fe, $120 \mathrm{mg}$; Cu, $5 \mathrm{mg}$; Mn, $60 \mathrm{mg}$; Zn, $25 \mathrm{~g}$; l, $0.3 \mathrm{mg}$; Se, $0.2 \mathrm{mg}$.

insights into the association between the microbial community in the cecum of Muscovy ducks and fat-related phenotypes.

\section{MATERIALS AND METHODS}

\section{Ducks and Sample Collection}

The Muscovy ducks were from Hewang Poultry Industry Co., Ltd. (Lanxi County, Jinhua City, Zhejiang Province, China). A population of 5,000 female ducks hatched on the same day were fed in cage-free pens on the plastic mesh floor with ad libitum water and diets under standardized conditions. Ducks received a commercial starter and finisher feed from 1 to 14 days old and 15 to 70 days old, respectively. The starter and finisher diets composition were stated in Table $\mathbf{1}$ according to previous studies $(17,18)$. The energy and nutrients levels met the estimated requirements for ducks (19). On day 70, a total of 200 healthy Muscovy ducks were randomly picked and weighed from the population of 5,000 ducks. All the 200 ducks were weighed and euthanized by $\mathrm{CO}_{2}$ asphyxiation. After being weighed and slaughtered, the birds were defeathered and opened to record the weight of abdominal fat tissues. The body weight, the eviscerated weight and the weight of the abdominal fat tissue were recorded promptly. The cecal contents were collected, frozen in liquid nitrogen immediately, and stored at $-80^{\circ} \mathrm{C}$ for DNA isolation. The abdominal fat percentage (AFP) was calculated according to the following formula

$$
\mathrm{AFP}=\frac{A F W}{A F W+E W} \times 100 \%
$$


where AFW denotes the abdominal fat weight and EW the eviscerated weight (20).

Eighty ducks with highest and lowest AFP were selected as high and low groups, respectively.

\section{DNA Extraction and Sequencing}

Genomic DNA was isolated from each cecal sample by a QIAamp DNA Stool Mini Kit (Qiagen, Valencia, CA) according to the manufacturers' instructions. The quality and concentration were evaluated using $1 \%$ agarose gel electrophoresis and a NanoDrop ND-1000 (Thermo Fisher Scientific, Waltham, MA, USA). High quality DNA was sequenced by next-generation sequencing (21). In detail, the barcode-fusion forward primer 515F (5'GTGCCAGCMGCCGCGGTAA-3') and the reverse primer 907R (5'- CCGTCAATTCMTTTRAGTTT-3') were employed to amplify the V4-V5 region of the bacterial 16S rRNA gene. The PCR conditions were as previously described (10). After PCR, amplicons were separated and qualified by using $2 \%$ (w/v) agarose gels. The DNA samples with $400-450$ bp bright bands between were chosen for further experiments. The DNA products were then purified by a GeneJET Gel Extraction Kit (Thermo Scientific) according to the manufacturer's instructions. An Illumina TruSeq DNA PCR-Free Library Preparation Kit (Illumina) was applied for sequencing library generation. The quality of the generated library was evaluated by using a Qubit 2.0 Fluorometer (Thermo Scientific) and an Agilent Bioanalyzer 2100 system. The qualified library was sequenced commercially by Mingke Biotechnology (Hangzhou) on an Illumina HiSeq platform, generating 250 bp paired-end read.

\section{Bioinformatics Analysis}

Illumina paired-end reads were demultiplexed and filtered in Quantitative Insights into Microbial Ecology (QIIME) quality filters (22) for clean reads, merged into tags using FLASH (23), and assigned to each sample in accordance with the unique barcode. The tags of each sample were analyzed after removing redundancies, and unique tags with $\geq 97 \%$ sequence similarity were assigned to the same operational taxonomic units (OTUs) using UPARSE and UCHIME. Selected OTUs were annotated with taxonomic information using the RDP classifier (24). Alphadiversity (Observed Species, Chao 1 estimator, ACE, Shannon, and Simpson indices) and beta-diversity were calculated and visualized in OriginLab 2018 (Northampton, MA, US).

\section{Identification of Adipogenesis-Related Microbiota}

To detect the microorganisms significantly related with fat deposition, the taxa present in $<30 \%$ of samples in Muscovy duck cecum were excluded. All the samples were successively sorted by host BW, AFW, and AFP and the relative abundance of each microorganism. The lowest $20 \%$ and highest $20 \%$ of the ranked birds were considered two distinct groups, and statistical analysis was performed for all the traits between the two groups. Furthermore, regression-based random forest models were performed to identify bacterial features associated with fat deposition in the randomForest package in the R project (25). Subsequently, the BW, AFW, and AFP were statically analyzed between the lowest $20 \%$ and highest $20 \%$ relative abundance of the common bacteria in fat-related bacterial characterization (detected in at least $30 \%$ of the cecal samples).

\section{Statistical Analysis}

Data are expressed as the mean \pm SEM. All statistical analyses were performed in OriginLab 2018. The difference between two groups was analyzed by unpaired two-tailed Student's $t$-test and considered significant when the $P$-value was no more than 0.05 .

\section{Accession Number}

The raw sequencing reads of this study have been deposited in NCBI under the accession number BioProject PRJNA663038.

\section{RESULTS}

\section{The Composition of the Cecal Microbiota in Muscovy Ducks}

To study the role of the cecal microbiota in adipose accumulation, we first investigated the composition of the cecal microbiota in Muscovy ducks. Cecal content samples from 200 Muscovy ducks were collected for bacterial 16S rRNA gene sequencing. A total of 7,469,466 high-quality sequence tags were generated and classified into 1,989 OTUs at the 97\% similarity level. At the phylum level, Bacteroidetes, Firmicutes, Fusobacteria, Cyanobacteria, Proteobacteria, and Deferribacteres were the top six phyla in the cecal contents, accounting for $96.78 \%$ of the total abundance (Figure 1). In detail, Bacteroidetes was the predominant phylum and accounted for $50.20 \%$ of the total sequences in the cecal content samples of Muscovy ducks, followed by Firmicutes, accounting for $37.45 \%$ of the total abundance. At the genus level, due to the richness of the microbiota, we listed only the top 10 genera in the cecal content of Muscovy ducks (Figure 1). Furthermore, the top four genera were Bacteroides, Oscillospiraceae_uncultured, Parabacteroides, and Bacteroidales_norank, accounting for $40.80 \%$ of the total bacteria in the cecum of Muscovy ducks.

\section{Duck Phenotype Characterization}

To characterize the distinct phenotype related to fat accumulation, we performed correlation analysis on the three phenotypes, namely, BW, AFW, and AFP. The observations on BW, AFW, and AFP are summarized in Table 2. AFW and AFP gave a high phenotypic correlation $\left(R^{2}=0.92, P<0.001\right.$, Figure 2A). However, the $R^{2}$ for the correlation between $\mathrm{BW}$ and AFW was 0.37 while the $R^{2}$ for the correlation between BW and AFP was 0.16.

To analyze how AFW and AFP were associated with each other, we compared ducks between the top and bottom $20 \%$ birds in terms of AFW and AFP. The 40 ducks with the highest and lowest AFW and AFP shared 34 and 32 individual birds, respectively, because of the high correlation between AFW and AFP (Figure 2B). Consequently, we set the highest and lowest $20 \%$ ducks in terms of AFP as the high and low groups, respectively. As shown in Figure 2, all of the AFP, AFW, and BW values gave highly significant differences between Muscovy ducks with the highest and lowest 20\% AFP $(n=40)$, suggesting the 

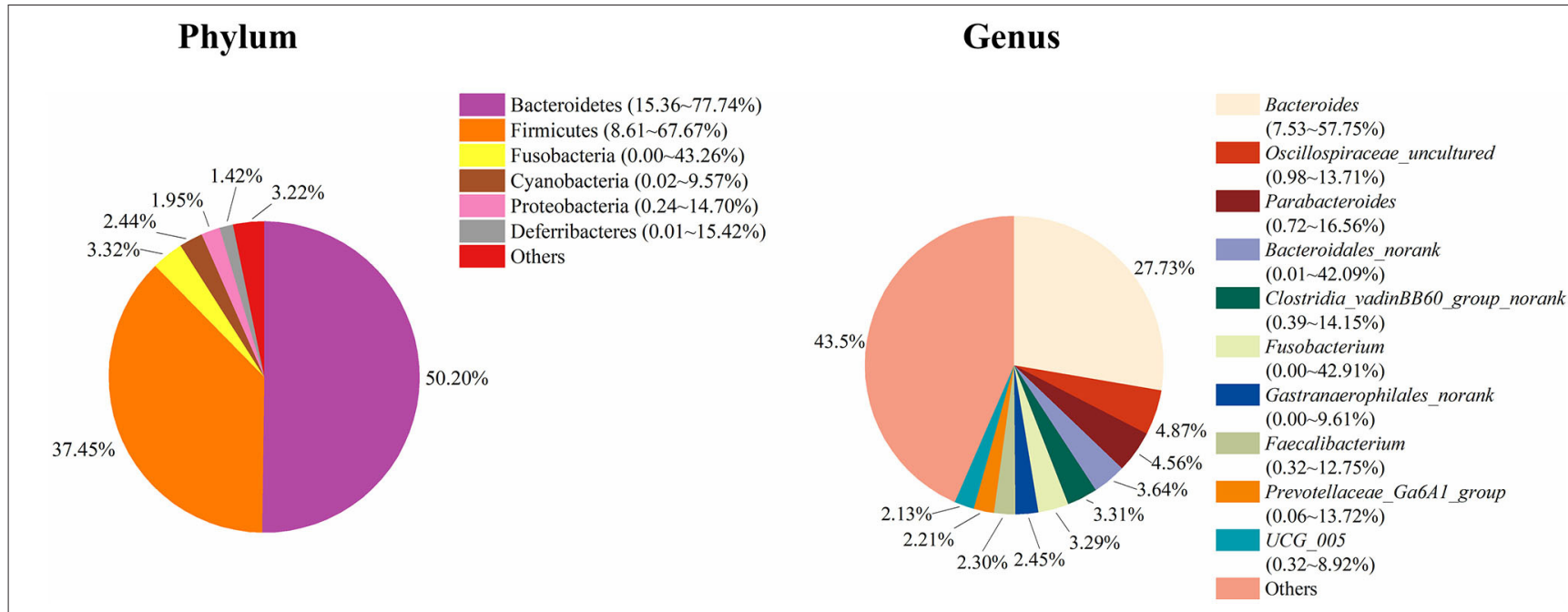

\section{Genus}

FIGURE 1 | The composition of the cecal microbiota in Muscovy ducks at the phylum and genus levels.

TABLE 2 | Descriptive statistics for Muscovy duck phenotypes.

\begin{tabular}{lrrrrrr}
\hline Traits & $\boldsymbol{N}$ & Mean & SD & CV (\%) & Maximum & Minimum \\
\hline BW (kg) & 200 & 2.80 & 0.26 & 9.12 & 3.58 & 1.97 \\
AFW (g) & 200 & 73.12 & 23.53 & 32.18 & 138.10 & 18.57 \\
AFP (\%) & 200 & 3.36 & 0.91 & 27.12 & 5.96 & 1.02 \\
\hline
\end{tabular}

$N$, the number of non-missing values; $S D$, standard deviation; $C V$, coefficient of variation; BW, body weight; AFW, abdominal fat weight; AFP, percentage of abdominal fat.

correlation of AFP with AFW and BW. To visualize the difference between the groups, we sorted the trait results by AFP and set the highest $20 \%(n=40)$ and the lowest $20 \%(n=40)$ as the high and low groups, respectively. As expected, with a significant difference $(P<0.001)$ in AFP, the AFW of ducks in the high and low groups showed a significant difference $(P<0.001$, Figure 3$)$.

\section{Differences in the Diversity, Composition and Potential Function of the Cecal Microbiota}

To further explore the differences in the $\alpha$-diversity and microbial composition between the high and low group Muscovy ducks, we compared the $\alpha$-diversity and bacterial composition in the cecum of the high and low group ducks. The $\alpha$-diversity of the cecal microbiota was measured by the Shannon index (Figure 4A), the Simpson index (Figure 4B), and the Chao index (Figure 4C). Overall, there was no significant difference in $\alpha$-diversity between the high and low groups $(P>0.05)$, suggestive of no significant difference in the microbial richness of the duck cecum in the high and low groups.

Next, we analyzed the microbial composition in the cecum of ducks in the high and low groups. The top six phyla in the cecal content of ducks in both the high and low groups were the same as those in the total group of 200 ducks, namely, Bacteroidetes, Firmicutes, Fusobacteria, Cyanobacteria,
Proteobacteria, and Deferribacteres with different relative abundances (Figure 5). The cecum of ducks in the high group had a relatively higher abundance of Firmicutes but a lower abundance of Bacteroidetes, accounting for 38.76 and $48.59 \%$, respectively. The relative abundances of the other four genera, Fusobacteria, Cyanobacteria, Proteobacteria, and Deferribacteres, were similar in the cecal samples in the high and low groups. At the genus level, Bacteroides, Parabacteroides, Oscillospiraceae_uncultured, Fusobacterium, Clostridia vadinBB60 group_norank, Gastranaerophilales_norank, UCG005, Faecalibacterium, Bacteroidales_norank, and Prevotellaceae Ga6A1 group composed the top 10 genera in both groups of ducks (Figure 5). Among them, Bacteroides was slightly less abundant in the low group ducks $(27.91 \%)$ than in the high group ducks (26.07\%). However, the relative abundance of Bacteroidales was higher in the low group ducks (5.40\%) than in the high group ducks $(2.21 \%)$. The proportions of the other genera were similar between the high and low group ducks.

\section{The Genera Treponema and Ruminococcus_torques_group are Significantly Associated with Fat Deposition}

It has previously been suggested that abdominal fat accumulation is associated with the gut microbiota (5). We further investigated which specific bacteria were linked with abdominal fat accumulation by running the randomForest analysis in the $\mathrm{R}$ project. The top 10 bacterial features predicting abdominal fat accumulation are listed in Figure 6. The common features in the lists of the top 10 AFW-related and AFP-related features were Treponema, Butyricicoccus, Ruminococcus_torques_group, Faecalicoccus, and Angelakisella. After adjusting the $P$-value to $P<0.05$, we focused on the two cecal genera, Treponema and Ruminococcus_torques_group, to further investigate how these two genera affected 

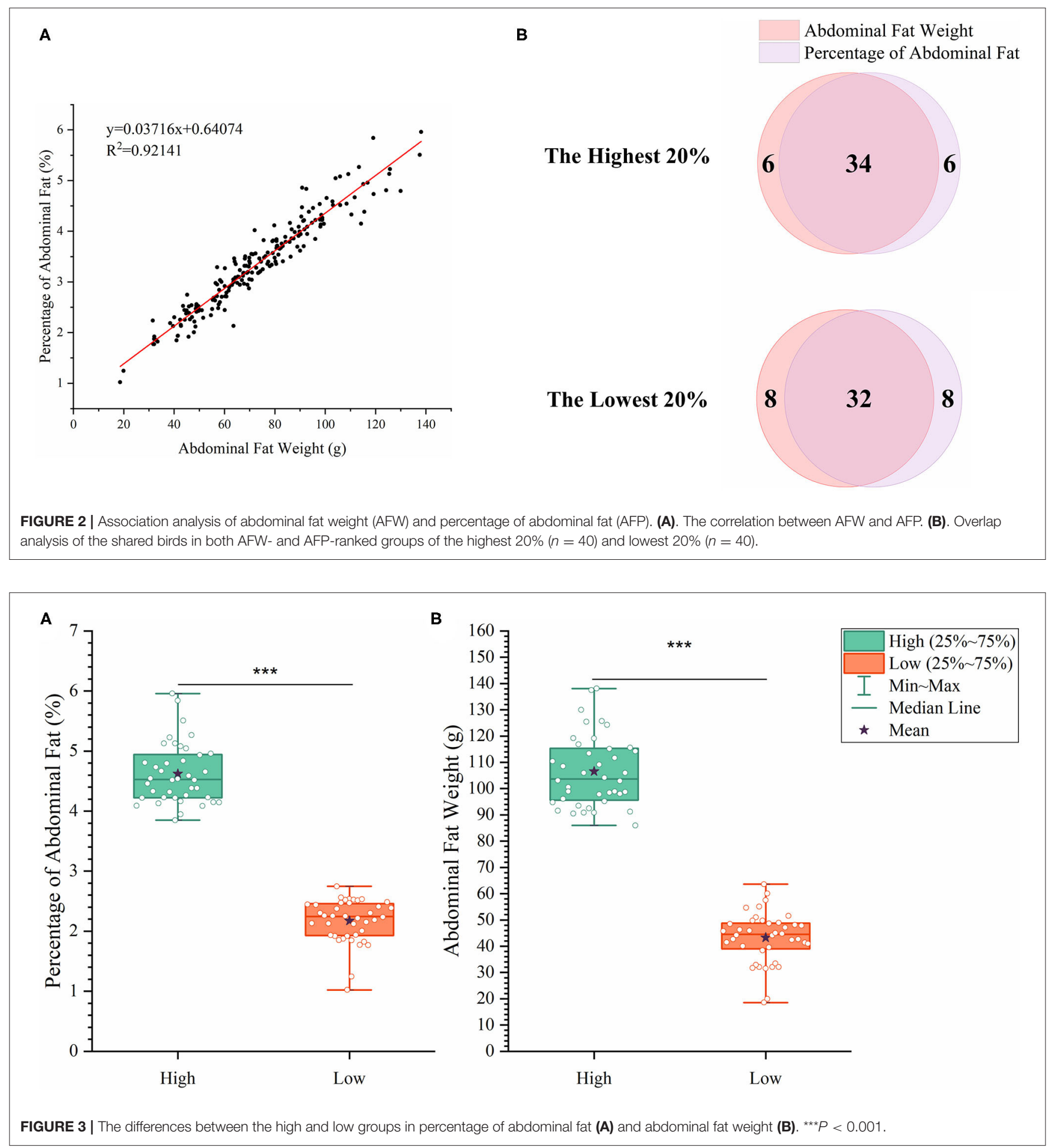

abdominal fat accumulation in Muscovy ducks. The ducks with Treponema and Ruminococcus_torques_group were sorted according to the relative abundances of these two genera. The 40 ducks with the most (20\%) and least (20\%) abundance of Treponema were selected for comparing the $\mathrm{AFW}, \mathrm{AFP}$, and BW. The same analysis was performed for
Ruminococcus_torques_group. As shown in Figure 7, Treponema and Ruminococcus_torques_group abundances were significantly related to AFW $(P<0.05)$. Furthermore, the $20 \%$ of ducks with the highest Treponema abundance tended to have a significantly lower AFP and BW than the $20 \%$ of ducks with the lowest Treponema abundance (Figure 7A, $P=0.056$ and 0.076), 

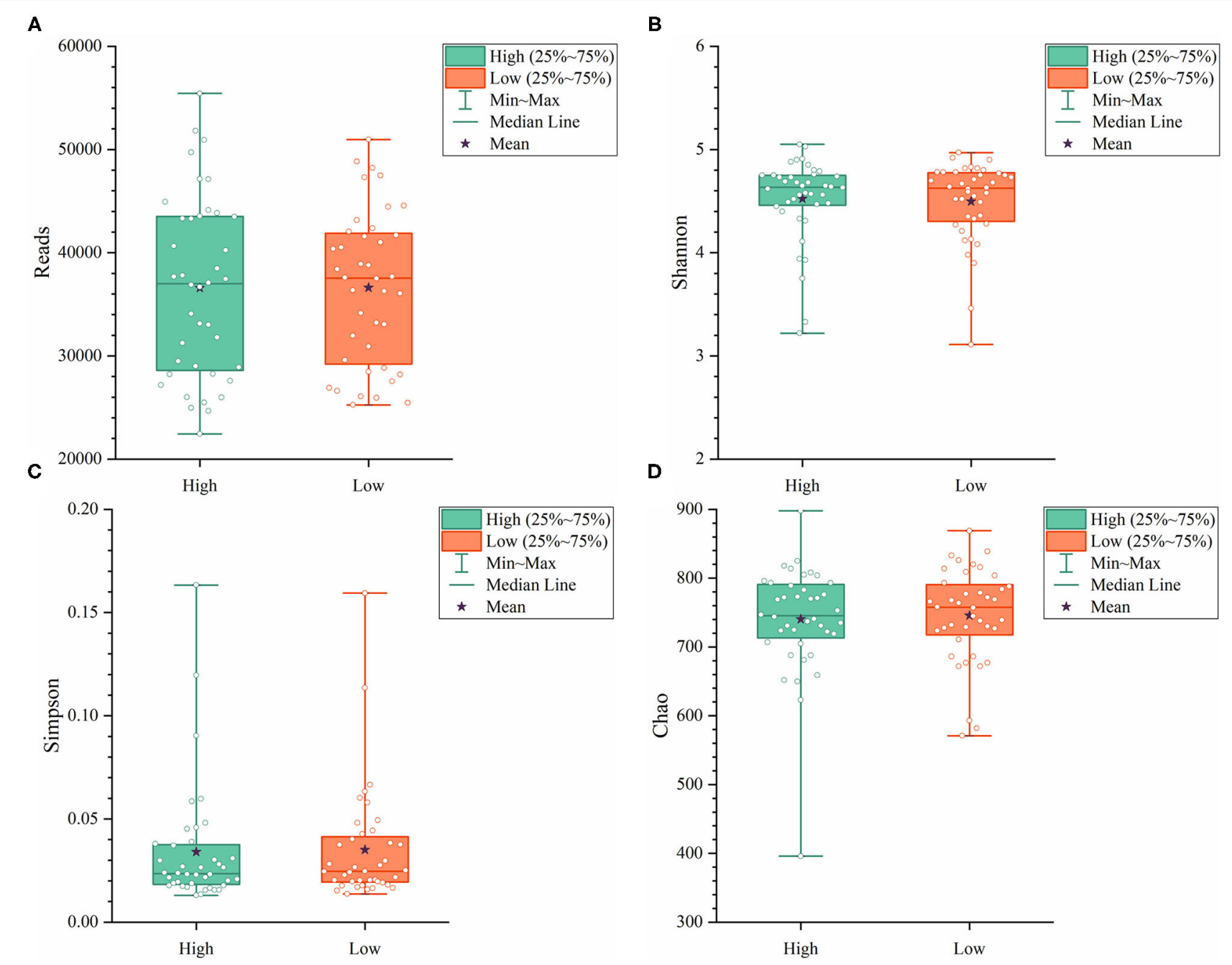

FIGURE 4 | The $\alpha$-diversity including Reads (A), Shannon (B), Simpson (C), and Chao (D) in the cecum of high and low group ducks.

indicating a negative correlation between fat accumulation and Treponema abundance. However, the opposite was true with the bacterial genus Ruminococcus_torques_group, giving a positive association between fat deposition and the relative abundance of Ruminococcus_torques_group (Figure 7B).

\section{DISCUSSION}

Muscovy ducks (Cairinamoschata) are characterized by meat quality with low fat and high protein content. The gut microbiota plays an essential role in energy metabolism and fat accumulation in the host and facilitates energy harvest from indigestible dietary fibers via fermentation $(8,27)$. Besides, the gut microbiota is involved in modulating the host immune system. The components of the gut microbiota vary according to the local microenvironment, such as oxygen gradients, $\mathrm{pH}$ levels, and nutrient availability along the gastrointestinal tract (5). Particularly in the cecum, Bacteroidetes and Firmicutes are the three predominant phyla in most animals. 16S rRNA sequencing results showed that the cecal bacteria are mainly composed of the Bacteroidetes and Firmicutes phyla in humans and mice (28), while Bacteroidetes and Firmicutes are the main phyla in the cecal digesta of pigs from farrow to finish $(29,30)$. Moreover, for chickens, Firmicutes, Bacteroides, Proteobacteria, and Actinobacteria account for more than $99 \%$ of the phyla in the cecum, where Firmicutes and Bacteroides are the most common ones $(31,32)$. Additionally, a study on Shaoxing ducks indicated that the most abundant phyla in the cecum were Firmicutes and Bacteroidetes (33). Not surprisingly, in the present study, we found that Bacteroidetes and Firmicutes were the two predominant phyla, while Bacteroides and Parabacteroides were the two most abundant genera in the cecum of Muscovy ducks (Figure 1). Due to the cecum being the distal portion of the GI tract, oxygen is limited, and indigestible dietary substrates are fermented in the cecum. Therefore, the bacterial components in the cecum are quite different from those in the small intestine. Due to fermentation mainly taking place in cecum, microbial 

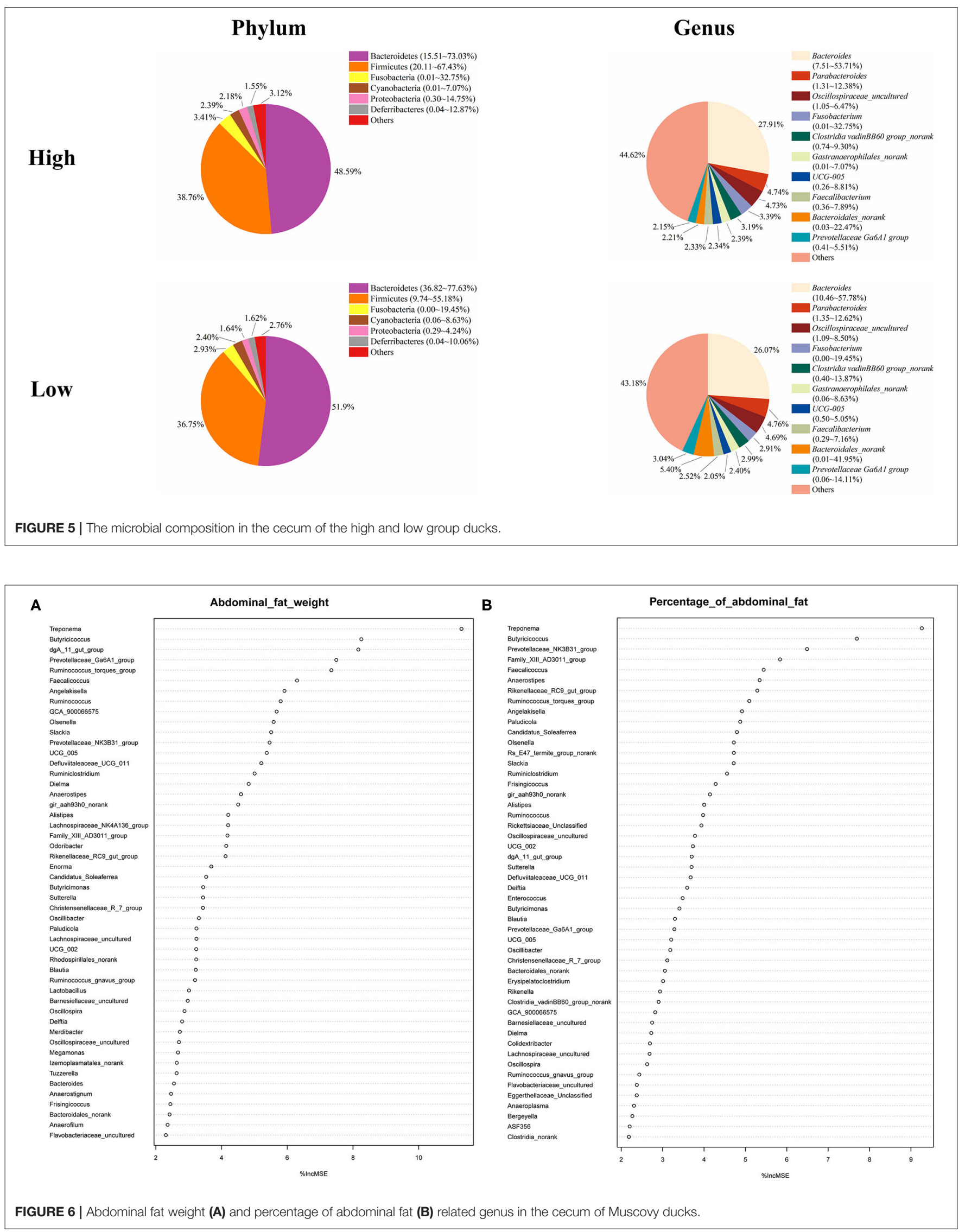


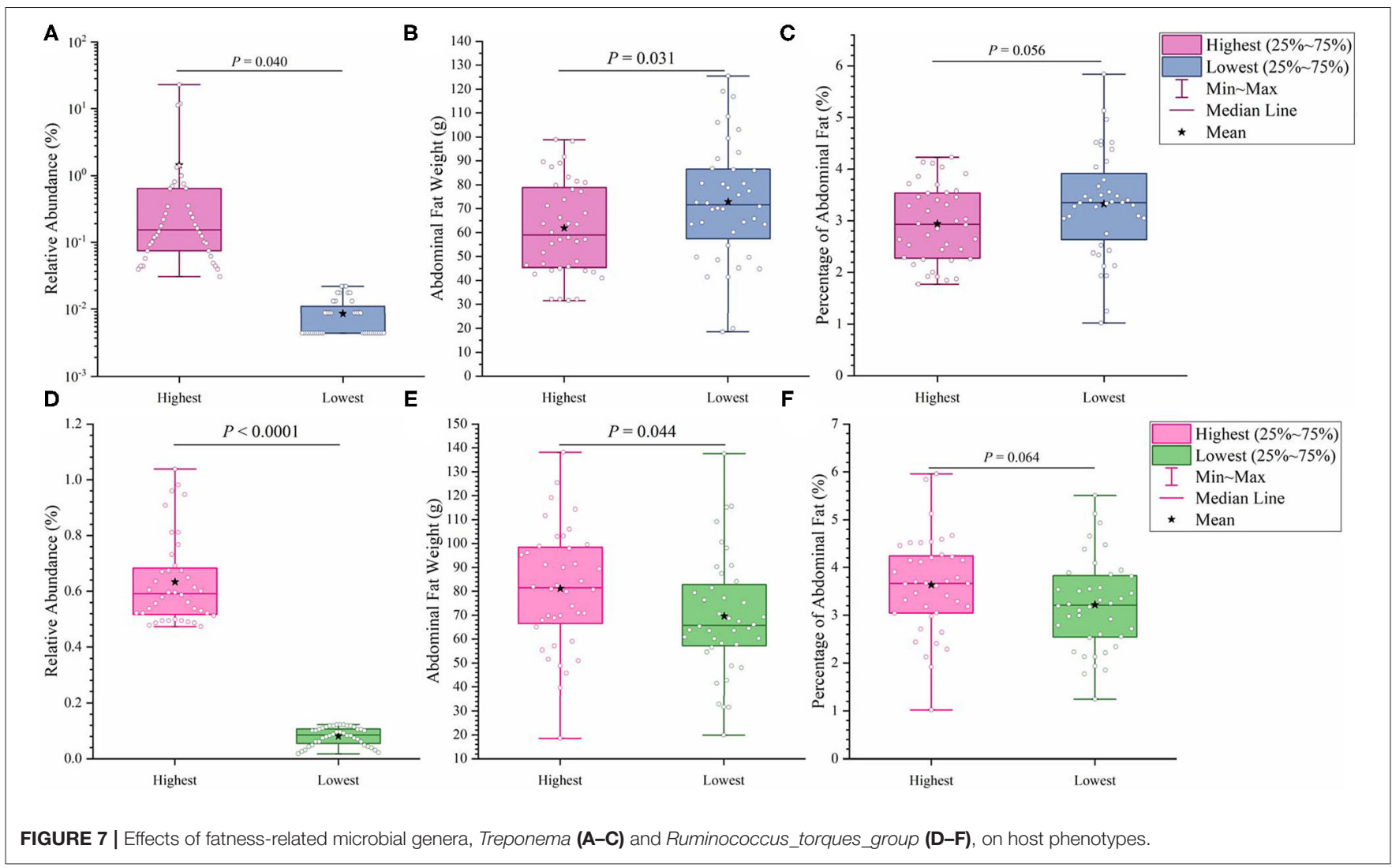

cells are most abundant in poultry cecum (34). The differences in bacterial component of duck cecum might explain a large portion of microbial regulating fat deposition, that's why we focus on the cecum in the present study. Furthermore, the majority of short chain fatty acids (SCFAs) are produced by bacteria in the cecum from indigestible substrate fermentation as the energy source for host cells as well as microorganisms in the cecum. With deepening microbiota investigations, increasing attention has been paid to the association between the gut microbiota and host energy metabolism and abdominal fat accumulation. The richness of these two phyla is considered to be correlated with the energy absorption from food by the host $(8,35)$. In the present study, compared to ducks in the low group, ducks in the high group gave a higher relative abundance of the Firmicutes phylum and a lower relative abundance of the Bacteroidetes phylum (Figure 5).

Among the top 10 AFW-related and AFP-related genera, Treponema and Ruminococcus_torques_group were found to be negatively and positively associated with fat deposition, respectively. With the ability to facilitate the absorption of energy from dietary indigestible polysaccharides (36), the abundance of Treponema groups is known to be negatively associated with fat content in the diet. With $99 \%$ identity of Treponema spp. with T. succinifaciens, Treponema spp. might contribute to host energy metabolism because T. succinifaciens is a carbohydrate metabolizer in the gut of termites, swine, and cattle (37). After the analysis of the gut microbiome in Japanese macaques, Prince et al. (38) found that long exposure to a high fat diet was strongly correlated with low Treponema abundance, suggestive of an association between high dietary fat content and low Treponema abundance. This result was confirmed by De Filippo et al. (39) who found that the Treponema abundance in leaner children from rural village (BR) and Nanoro town (BT) was significantly higher than that in children from the capital city of Burkina Faso (BC) and Europe (EU). Additionally, from 300 porcine cecum lumen samples, association analysis showed that Treponema was one of the most negatively intramuscular fat-associated bacteria (40), providing additional evidence that Treponema is involved in fat deposition with a negative correlation. Consistently, we observed that the high Treponema abundance group had a significantly lower AFW than the low Treponema abundance group $(P<0.05)$. The AFP of ducks in the high Treponema abundance group tended be lower than that of ducks in the low Treponema abundance group $(P<0.01$; Figure 7$)$.

Ruminococcus_torques_groupis a genera derived from genus Mediterraneibacter from the family Lachnospiraceae (NCBI Taxonomy Browser, https://www.ncbi.nlm.nih.gov/ Taxonomy/Browser/wwwtax.cgi) and was discovered to ferment gastric mucins and identified as a butyrate-producing bacterium (41). A study on Fubrick tea aqueous extract (FTEs) in mice indicated that FTEs were able to alleviate visceral fat deposition via an increase in the abundance of Lachnospiraceae (42). This result suggests a positive correlation between fat deposition and Lachnospiraceae 
abundance. A similar positive association of abdominal fat and subcutaneous fat thickness with Lachnospiraceae abundance was observed in broilers (43). Because it is a species of the family Lachnospiraceae, the abundance of Ruminococcus_torques_group is supposed to be positively related to fat accumulation. Besides, a randomized, doubleblinded and crossover design clinical trial on resistant starch showed that a low level of Ruminococcus_torques_group would assist in reducing the body fat (44), suggestive of a positive relationship between the Ruminococcus_torques_group level and fat accumulation. Additionally, Ruminococcus_torques_group, categorized as Clostridium cluster XIVa, mainly produces butyrate (45). Butyrate-producing bacteria were found to promote fat deposition (46) because they are able to convert dietary fiber to butyrate by fermentation to provide additional energy to the host (26). In our study, the ducks with high Ruminococcus_torques_group abundance showed significantly higher AFW $(P<0.05)$ and a tendency toward higher AFP $(P<0.01)$ than the ducks with low Ruminococcus_torques_group abundance (Figure 7B). No significant difference was observed in BW between the high and low Ruminococcus_torques_group abundance groups. Our result and previous studies illustrate the positive correlation of the Ruminococcus_torques_group level with fat deposition.

Collectively, we thoroughly investigated the Muscovy duck cecal microbiota by $16 \mathrm{~S}$ rRNA sequencing and analyzed the correlation of duck fat deposition with the cecal microbiota. It is not surprising that various bacteria existed in the duck cecum with the dominant phyla being Bacteroidetes and Firmicutes, giving a result similar to that with other duck species. The variability in fat deposition of a duck population was correlated with the cecal microbiota in Muscovy ducks. Interestingly, the genera Treponema and Ruminococcus_torques_group were negatively and positively associated with fat deposition, respectively. These results would provide basic evidence that the cecal microbiota contributes to regulating fat deposition and is beneficial to the

\section{REFERENCES}

1. Srivastava G, Apovian CM. Current pharmacotherapy for obesity. Nat Rev Endocrinol. (2018) 14:12-24. doi: 10.1038/nrendo.201 7.122

2. Chen S, Xiang H, Zhang H, Zhu X, Wang D, Wang J, et al. Rearing system causes changes of behavior, microbiome, and gene expression of chickens. Poult Sci. (2019) 98:3365-76. doi: 10.3382/ps/pez140

3. Siegel PB. Evolution of the modern broiler and feed efficiency. Annu Rev Anim Biosci. (2014) 2:375-85. doi: 10.1146/annurev-animal-022513-114132

4. Wen C, Yan W, Zheng J, Ji C, Zhang D, Sun C, et al. Feed efficiency measures and their relationships with production and meat quality traits in slower growing broilers. Poult Sci. (2018) 97:2356-64. doi: 10.3382/ps/pey062

5. Wen C, Yan W, Sun C, Ji C, Zhou Q, Zhang D, et al. The gut microbiota is largely independent of host genetics in regulating fat deposition in chickens. ISME J. (2019) 13:1422-36. doi: 10.1038/s41396-019-0367-2

6. Janssen AW, Kersten S. The role of the gut microbiota in metabolic health. FASEB J. (2015) 29:3111-23. doi: 10.1096/fj.14-269514

7. Gérard P. Gut microbiota and obesity. Cell Mol Life Sci. (2016) 73:147-62. doi: 10.1007/s00018-015-2061-5 development of strategies for abdominal fat reduction in the duck industry.

\section{DATA AVAILABILITY STATEMENT}

The datasets generated for this study can be found in online repositories. The names of the repository/repositories and accession number(s) can be found below: https://www.ncbi.nlm. nih.gov/, BioProject PRJNA663038.

\section{ETHICS STATEMENT}

The animal study was reviewed and approved by Animal Care and Use Committee of Zhejiang Academy of Agricultural Sciences.

\section{AUTHOR CONTRIBUTIONS}

WL, XL, LL, BD, and WW: conceptualization, methodology, and experiment performance. WL: data analysis, writing-original draft preparation. HY and YX: writing-review and editing. All authors have read and agreed to the published version of the manuscript.

\section{FUNDING}

This work was supported by the State Key Laboratory for Managing Biotic and Chemical Threats to the Quality and Safety of Agro-products, Zhejiang Academy of Agricultural Sciences (2010DS700124-ZZ1905), and the National Waterfowl Industry Technology System of China (CARS-42-27).

\section{ACKNOWLEDGMENTS}

This was a short text to acknowledge the contributions of specific colleagues, institutions, or agencies that aided the efforts of the authors.

8. Turnbaugh PJ, Ley RE, Mahowald MA, Magrini V, Mardis ER, Gordon JI. An obesity-associated gut microbiome with increased capacity for energy harvest. Nature. (2006) 444:1027-31. doi: 10.1038/nature05414

9. Ridaura VK, Faith JJ, Rey FE, Cheng J, Duncan AE, Kau AL, et al. Gut microbiota from twins discordant for obesity modulate metabolism in mice. Science. (2013) 341:1241214. doi: 10.1126/science. 1241214

10. Yang H, Xiang Y, Robinson K, Wang J, Zhang G, Zhao J, et al. Gut microbiota is a major contributor to adiposity in pigs. Front Microbiol. (2018) 9:3045. doi: 10.3389/fmicb.2018.03045

11. Kokoszyński D, Wilkanowska A, Arpášová H, Hrnčár C. Comparison of some meat quality and liver characteristics in Muscovy and mule ducks. Arch Anim Breed. (2020) 63:137-44. doi: 10.5194/aab-63-137-2020

12. Wang J, Huang Y, Ling J, Wang Z, Zhu G. Transfection of embryonated Muscovy duck eggs with a recombinant plasmid is suitable for rescue of infectious Muscovy duck parvovirus. Arch Virol. (2017) 162:3869-74. doi: 10.1007/s00705-017-3541-8

13. Chen S, Lin F, Chen S, Hu Q, Cheng X, Jiang B, et al. Development of a live attenuated vaccine against Muscovy duck reovirus infection. Vaccine. (2018) 36:8001-7. doi: 10.1016/j.vaccine.2018.10.102 
14. Liu Z, Li M, Yan P, Zhu Z, Liao L, Chen Q, et al. Transcriptome analysis of the effects of Hericium erinaceus polysaccharide on the lymphocyte homing in Muscovy duck reovirus-infected ducklings. Int J Biol Macromol. (2019) 140:697-708. doi: 10.1016/j.ijbiomac.2019.08.130

15. Wan C, Cheng L, Chen C, Liu R, Shi S, Fu G, et al. A duplex PCR assay for the simultaneous detection and differentiation of Muscovy duck parvovirus and goose parvovirus. Mol Cell Probe. (2019) 47:101439. doi: 10.1016/j.mcp.2019.101439

16. Wang Q, Liu M, Chen Y, Xu L, Wu B, Wu Y, et al. Muscovy duck reovirus p10.8 protein induces ER stress and apoptosis through the Bip/IRE1/XBP1 pathway. Vet Microbiol. (2019) 228:234-45. doi: 10.1016/j.vetmic.2018. 12.011

17. Kong C, Adeola O. Additivity of amino acid digestibility in corn and soybean meal for broiler chickens and White Pekin ducks. Poult Sci. (2013) 92:2381-8. doi: 10.3382/ps.2013-03179

18. Yang H, Lyu W, Lu L, Shi X, Li N, Wang W, et al. Biogeography of microbiome and short-chain fatty acids in the gastrointestinal tract of duck. Poult Sci. (2020) 99:4016-27. doi: 10.1016/j.psj.2020.03.040

19. National Research Council. Nutrient Requirements of Poultry. 9th revised ed, Washington, DC: The National Academies Press (1994).

20. NY/T 823. Performance Terminology and Measurements for Poultry. Beijing: The Ministry of Agriculture of the People's Republic of China (2020). (In Chinese).

21. Xiao Y, Kong F, Xiang Y, Zhou W, Wang J, Yang H, et al. Comparative biogeography of the gut microbiome between Jinhua and Landrace pigs. Sci Rep. (2018) 8:5985. doi: 10.1038/s41598-018-24289-z

22. Lawley B, Tannock GW. Analysis of $16 \mathrm{~S}$ rRNA gene amplicon sequences using the QIIME software package. Methods Mol Biol. (2017) 1537:153-63. doi: 10.1007/978-1-4939-6685-1_9

23. Magoc T, Salzberg SL. FLASH: fast length adjustment of short reads to improve genome assemblies. Bioinformatics. (2011) 27:2957-63. doi: 10.1093/bioinformatics/btr507

24. Wang Q, Garrity GM, Tiedje JM, Cole JR. Naive Bayesian classifier for rapid assignment of rRNA sequences into the new bacterial taxonomy. Appl Environ Microbiol. (2007) 73:5261-7. doi: 10.1128/AEM. 00062-07

25. Wang X, Tsai T, Deng F, Wei X, Chai J, Knapp J, et al. Longitudinal investigation of the swine gut microbiome from birth to market reveals stage and growth performance associated bacteria. Microbiome. (2019) 7:109. doi: 10.1186/s40168-019-0721-7

26. Blaut M. Gut microbiota and energy balance: role in obesity. Proc Nutr Soc. (2015) 74:227-34. doi: 10.1017/S0029665114001700

27. Yang H, Xiao Y, Wang J, Xiang Y, Gong Y, Wen X, et al. Core gut microbiota in Jinhua pigs and its correlation with strain, farm and weaning age. J Microbiol. (2018) 56:346-55. doi: 10.1007/s12275-018-7486-8

28. Bradlow HL. Obesity and the gut microbiome: pathophysiological aspects. Horm Mol Biol Clin Investig. (2014) 17:53-61. doi: 10.1515/hmbci-20 13-0063

29. Yang H, Huang X, Fang S, Xin W, Huang L, Chen C. Uncovering the composition of microbial community structure and metagenomics among three gut locations in pigs with distinct fatness. Sci Rep. (2016) 6:27427. doi: $10.1038 /$ srep 27427

30. De Rodas B, Youmans BP, Danzeisen JL, Tran H, Johnson TJ. Microbiome profiling of commercial pigs from farrow to finish. J Anim Sci. (2018) 96:177894. doi: 10.1093/jas/sky109

31. Oakley BB, Lillehoj HS, Kogut MH, Kim WK, Maurer JJ, Pedroso A, et al. The chicken gastrointestinal microbiome. FEMS Microbiol Lett. (2014) 360:10012. doi: 10.1111/1574-6968.12608

32. Robinson K, Becker S, Xiao Y, Lyu W, Yang Q, Zhu H, et al. Differential impact of subtherapeutic antibiotics and ionophores on intestinal microbiota of broilers. Microorganisms. (2019) 7:282. doi: 10.3390/microorganisms70 90282
33. Wang S, Chen L, He M, Shen J, Li G, Tao Z, et al. Different rearing conditions alter gut microbiota composition and host physiology in Shaoxing ducks. Sci Rep. (2018) 8:7387. doi: 10.1038/s41598-018-25760-7

34. Glendinning L, Stewart RD, Pallen MJ, Watson KA, Watson M. Assembly of hundreds of novel bacterial genomes from the chicken caecum. Genome Biol. (2020) 21:34. doi: 10.1186/s13059-020-1947-1

35. Ley RE, Bäckhed F, Turnbaugh P, Lozupone CA, Knight RD, Gordon JI. Obesity alters gut microbial ecology. Proc Natl Acad Sci U S A. (2005) 102:11070-5. doi: 10.1073/pnas.0504978102

36. De Filippo C, Cavalieri D, Di Paola M, Ramazzotti M, Poullet JB, Massart S, et al. Impact of diet in shaping gut microbiota revealed by a comparative study in children from Europe and rural Africa. Proc Natl Acad Sci U S A. (2010) 107:14691-6. doi: 10.1073/pnas.1005963107

37. Obregon-Tito AJ, Tito RY, Metcalf J, Sankaranarayanan K, Clemente JC, Ursell LK, et al. Subsistence strategies in traditional societies distinguish gut microbiomes. Nat Commun. (2015) 6:6505. doi: 10.1038/ncomms7505

38. Prince AL, Pace RM, Dean T, Takahashi D, Kievit P, Friedman JE, et al. The development and ecology of the Japanese macaque gut microbiome from weaning to early adolescence in association with diet. Am J Primatol. (2019) 81:e22980. doi: 10.1002/ajp.22980

39. De Filippo C, Di Paola M, Ramazzotti M, Albanese D, Pieraccini G, Banci E, et al. Diet, environments, and gut microbiota. A preliminary investigation in children living in Rural and Urban Burkina Faso and Italy. Front Microbiol. (2017) 8:1979. doi: 10.3389/fmicb.2017.01979

40. Fang S, Xiong X, Su Y, Huang L, Chen C. 16S rRNA gene-based association study identified microbial taxa associated with pork intramuscular fat content in feces and cecum lumen. BMC Microbiol. (2017) 17:162. doi: 10.1186/s12866-017-1055-x

41. Salyers AA, West SE, Vercellotti JR, Wilkins TD. Fermentation of mucins and plant polysaccharides by anaerobic bacteria from the human colon. Appl Environ Microbiol. (1977) 34:529-33. doi: 10.1128/AEM.34.5.529-533.1977

42. Jing N, Liu X, Jin M, Yang X, Hu X, Li C, et al. Fubrick tea attenuates high-fat diet induced fat deposition and metabolic disorder by regulating gut microbiota and caffeine metabolism. Food Funct. (2020) 11:6971-86. doi: 10.1039/D0FO01282C

43. Niu J, Zhang J, Wei L, Ma X, Zhang W, Nie C. Cottonseed meal fermented by Candida tropical reduces the fat deposition in white-feather broilers through cecum bacteria-host metabolic cross-talk. Appl Microbiol Biotechnol. (2020) 104:4345-57. doi: 10.1007/s00253-020-10538-7

44. Zhang L, Ouyang Y, Li H, Shen L, Ni Y, Fang Q, et al. Metabolic phenotypes and the gut microbiota in response to dietary resistant starch type 2 in normal-weight subjects: a randomized crossover trial. Sci Rep. (2019) 9:4736. doi: 10.1038/s41598-018-38216-9

45. De Cesare A, Sirri F, Manfreda G, Moniaci P, Giardini A, Zampiga M, et al. Effect of dietary supplementation with Lactobacillus acidophilus D2/CSL (CECT 4529) on caecum microbioma and productive performance in broiler chickens. PLoS ONE. (2017) 12:e0176309. doi: 10.1371/journal.pone.0176309

46. Yang X, Zhang B, Guo Y, Jiao P, Long F. Effects of dietary lipids and Clostridium butyricum on fat deposition and meat quality of broiler chickens. Poult Sci. (2010) 89:254-60. doi: 10.3382/ps.2009-00234

Conflict of Interest: The authors declare that the research was conducted in the absence of any commercial or financial relationships that could be construed as a potential conflict of interest.

Copyright (C) 2021 Lyu, Liu, Lu, Dai, Wang, Yang and Xiao. This is an open-access article distributed under the terms of the Creative Commons Attribution License (CC $B Y)$. The use, distribution or reproduction in other forums is permitted, provided the original author(s) and the copyright owner(s) are credited and that the original publication in this journal is cited, in accordance with accepted academic practice. No use, distribution or reproduction is permitted which does not comply with these terms. 